\title{
Sulforaphane alter the microbiota and mitigate colitis severity on mice ulcerative colitis induced by DSS
}

Yan Zhang ${ }^{1 \dagger}$, Luxuan Tan ${ }^{1,2+}$, Chao Li ${ }^{1}$, Han Wu$^{1}$, Dan Ran ${ }^{1}$ and Zhenyu Zhang ${ }^{1 *}$ (i)

\begin{abstract}
Sulforaphane (SFN) is a kind of natural isothiocyanate, which exists in cruciferous plants. Only few studies were about the anti-inflammatory effects of sulforaphane in ulcerative colitis. In this study, our purpose is to explore the effects of sulforaphane on the intestinal microbial community of UC mice. The severity of mice colitis were measured by colon length, survial rate, body weight and disease activity index (DAI) score. Histological and morphological evaluation of colon tissues were performed by HE. 16S rRNA gene amplicon pyrosequencing was used to analyza the changes of mouse flora. The variety of flora expression were explored using quantitative PCR. Sulforaphane treated mice had larger body weight and longer colon length than DSS-induced mice. The colon tissues of DSS group showed congestion and edema. Meanwhile, treatment with sulforaphane effectively reducted the damage scores and MPO activity. Sulforaphane reversed DSS-induced gut dysbiosis. Sulforaphane would shift the balance to Butyricicoccus on inflammation. The possible anti-inflammatory mechanism of sulforaphane is to coordinate with the probiotics such as Butyricicoccus. In summary, these findings proved that sulforaphane might be a useful content and serve as a potential therapy in the treatment of UC.
\end{abstract}

Keywords: Sulforaphane, Gut dysbiosis, Ulcerative colitis

\section{Introduction}

Ulcerative colitis (UC) is a kind of chronic nonspecific inflammatory bowel disease. Epidemiological studies shown that the prevalence of UC in the world is 5.5024.30 in 100,000. In North America and Europe, the prevalence of UC is higher, about 24.30 in 100,000 and 19.20 in 100,000 respectively. In Asia and the Middle East, about 6.30 in 100,000 (Ng et al. 2013). Until now, the etiology of UC is unclear. It can be related to heredity, geographical environment, dietary habits, autoimmunity, smoking or other factors.

Recently, more and more studies have shown that intestinal flora has a high correlation to the occurrence

\footnotetext{
*Correspondence: zzy6565@sina.com

†Yan Zhang and Luxuan Tan contributed equally

1 Department of Gastroenterology, Nanjing First Hospital, Nanjing

Medical University, 68 Changle Road, Nanjing 210006, China

Full list of author information is available at the end of the article
}

and development of UC. Many factors, espicially diet and environment, can react to intestinal flora and then causes flora imbalance (Guinane and Cotter 2013; Goto et al. 2015). Normally, intestinal flora would not cause colonic mucosal inflammation directly. When the host is in a stressful environment or some pathological conditions, harmful bacteria or opportunistic pathogens in the intestine have a higher-risk to colonize, invade the intestinal epithelium and cause inflammation (Yang et al. 2017). However, the causal relationship between intestinal flora imbalance and UC remains controversial (Pei et al. 2019).

Sulforaphane, the main hydrolytic active product of glucosinolates, belongs to the isothiocyanate family and widely exists in cruciferous vegetables such as broccoli, kale and Northern carrot. Broccoli, especially 3-day-old broccoli buds, contains a high concentration of sulforaphane, which is the main source of substances in our clinical research (Liu et al. 2008). Wagner et al.
Springer Open

(c) The Author(s) 2020. This article is licensed under a Creative Commons Attribution 4.0 International License, which permits use, sharing, adaptation, distribution and reproduction in any medium or format, as long as you give appropriate credit to the original author(s) and the source, provide a link to the Creative Commons licence, and indicate if changes were made. The images or other third party material in this article are included in the article's Creative Commons licence, unless indicated otherwise in a credit line to the material. If material is not included in the article's Creative Commons licence and your intended use is not permitted by statutory regulation or exceeds the permitted use, you will need to obtain permission directly from the copyright holder. To view a copy of this licence, visit http://creativecommons.org/licenses/by/4.0/. 
(2013) established an animal model of DSS-induced UC which is pretreated with SFN at a dose of $25 \mathrm{mg} /$ $\mathrm{kg}$. The results showed that SFN could alleviate diarrhea, weight loss and colon tissue damage in mice, and reduced inflammatory biomarkers such as interleukin- 6 and interferon-gamma. In mdrla (-/-) IBD mice, SFN-rich fresh broccoli diet has been shown to alter intestinal microflora and alleviate colitis (Paturi et al. 2012).

However, the effect of sulforaphane on intestinal flora is unclear. Therefore, a DSS-induced UC mice model used to study the intervention of SFN on UC and observe the effect of SFN on intestinal flora, in order to provide new ideas for the treatment of ulcerative colitis.

\section{Materials and methods}

\section{Animals and grouping}

18 healthy male C57BL/6 (18-22 g) were purchased from the laboratory animal center of Nanjing medical university. Randomly divided animals into three equal groups, including the dextran sulfate sodium induced group (called DSS group below), SFN group, and blank group. In 2 weeks, SFN group was given SFN intragastric administration $20 \mathrm{mg} / \mathrm{kg} /$ days, other two groups were given distilled normal saline. But start from the second week, SFN group and DSS group were administrated 2.5\% DSS in drinking water to induce UC additionly. Body weight was measured daily at the same time. At the end of the experient, sacrifice the mice by cervical dislocation and then collect the colon specimens. L-Sulforaphane was gift from Huahan Biotechnology Co.Ltd, Ganzhou, Jiangxi, China (batch number: 20180101).

\section{Histological and morphological evaluation}

Colon tissues were preserved in $10 \%$ neutral formalin. Then embedded the tissues in paraffin and stained with hematoxylin and eosin (HE). A standard histological scoring system was used as a criterion to evaluate colitis. Briefly, $0=$ no damage; $1=$ extent of disease $<25 \%$; $2=$ extent of disease $26-50 \%, 3=$ extent of disease $51-75 \%, 4=$ extent of disease $>75 \%$.

\section{UC severity evaluation}

UC severity evaluation was based on the disease activity index (DAI) score which has been widely used in animal models. The researcher recorded and scored the changes following the protocol, which contained hemoccult positivity, weight, stool consistency and gross bleeding. Then all these parameters combined to DAI score for evaluation.

\section{$16 S$ rRNA gene amplicon pyrosequencing}

Collected fecal samples and extracted DNA with using the Stool DNA Kit (Omega Bio-tek, Norcross, GA, U.S) according to the manufacturer protocols. Using spectrophotometer and $1 \%$ agarose gel electrophoresis to measure the purity and concentration of microbial DNA. Next step was the V4-V5 region of the bacteria 16S rRNA gene amplicon, inwhere two universal primers 338F (ACTAAT ACGGAGGCAGCAG) and 806R (GGACTACNNGGG TATCTAAT) was used by PCR. The first step of PCR was $94{ }^{\circ} \mathrm{C}$ for $5 \mathrm{~min}$, then did 28 cycles of $94{ }^{\circ} \mathrm{C}$ for $30 \mathrm{~s}, 55^{\circ} \mathrm{C}$ for $30 \mathrm{~s}$ and $72{ }^{\circ} \mathrm{C}$ for $60 \mathrm{~s}$, the final step was $72{ }^{\circ} \mathrm{C}$ for 7 min. Each PCR reaction system was consisted of $2 \times$ Taq Plus Master Mix $(12.5 \mu \mathrm{L})$, BSA $(3 \mu \mathrm{L}$ with $2 \mathrm{ng} / \mu \mathrm{l}$ density), $1.0 \mu \mathrm{L}$ of each primer $(5 \mu \mathrm{M}), \mathrm{dd}_{2} \mathrm{O}$ and $30 \mathrm{ng}$ of template DNA in a $25 \mu \mathrm{l}$ volume. PCR products were collected and purified with QIAquick Gel Extraction Kit (QIAGEN, Germany).

Pooled purified amplicons at equimolar ratios, then paired-end sequenced $(2 \times 250)$ on an Illumina MiSeq platform. The raw data were stored in the NCBI Sequence Read Archive (SRA) database. Using Trimmomatic to demultiplex and qualityfilter the raw fast files, and using silva to cluster Operational Taxonomic Units (OTUs) with 97\% similarity cutoff. Then UCHIME was used to identify and remove chimeric sequences. The silva (SSU115) 16S rRNA database was analysed by RDP Classifier (http://rdp.cme.msu.edu/) with a confidence threshold of 70\%. At each taxonomical level (Phylum, Class, Order, Family, and Genus), one analysis was performed respectively. The diversity of alpha (within samples) and beta (among samples) were analyzed by using Inhouse Perl scripts. The $\alpha$ diversity index was calculated by using Mothur software (version 1.31.2). The R (v3.1.1) software package is used for clustering analysis. UniFrac algorithm uses the information of system evolution to compare the differences of bacteria groups among samples, and makes further statistical analysis of the results.

\section{Quantitative PCR of butyricicoccus in fecal DNA of mice}

Using Trizol (Invitrogen, USA) to extract the total RNA in colon tissue, and using RNA reverse transcription kits to reverse-transcrib RNA into cDNA. Upstream primer 5'-ACCTGAAGAATAAGCTCC-3', Downstream primer 5'-GATAACGCTTGCTCCCTACGT-3'. Using a SYBR Premix EX Taq Realtime PCR Master Mix (TaKaRa) to check mRNA expression levels on a Bio-Rad Q5 instrument (Bio-Rad, CA, USA).

\section{Statistical analysis}

Data collected from all three groups were represented as the mean \pm standard error of the mean (SEM). More than 
two groups' datasets were evaluated by one-way ANOVA followed by Newman-Keuls post hoc tests. Among all three groups, statistical significance of nonparametric variables' differences was assessed by the nonparametric Turkey test followed by the Mann-Whitney U test when $\mathrm{P}<0.05$. The statistical analysis were performed by SPSS 22.0 software (Chicago, IL, USA).

\section{Results}

\section{Effects of sulforaphane on UC clinical symptoms} in DSS-induced mice

Mice in the blank group had normal diet, activity, stool consistency, hair grown and weight gain. However, in the DSS group, the mice showed a obvious decrease in body weight and colon length (Fig. 1a, c, d). Moreover, as expected, the DAI scores of DSS-induced mice increased significantly (Fig. 1b). These mice showed anorexia, reduced activity, loose stool, drab hair color and weight loss.

Compared to the blank group, DSS-induced mice had obvious weight loss and lower DAI scores. The symptoms in the SFN group were milder than the DSS group.

\section{Histological observation and evaluation}

The estimation of damage extent of colon tissues was showed in histological analysis. HE staining of blank group showed normal colonic mucosal epithelium (Fig. 2a). Instead, DSS group had extensive glandular destruction and inflammatory cell infiltration in the submucosa. Compared with DSS group, SFN group showed milder degree of congestion and edema. As shown in Fig. 2b, c, SFN group and DSS group had a signifcant difference between the microscopic damage scores and MPO activity $(\mathrm{P}<0.05)$. Moreover, the difference
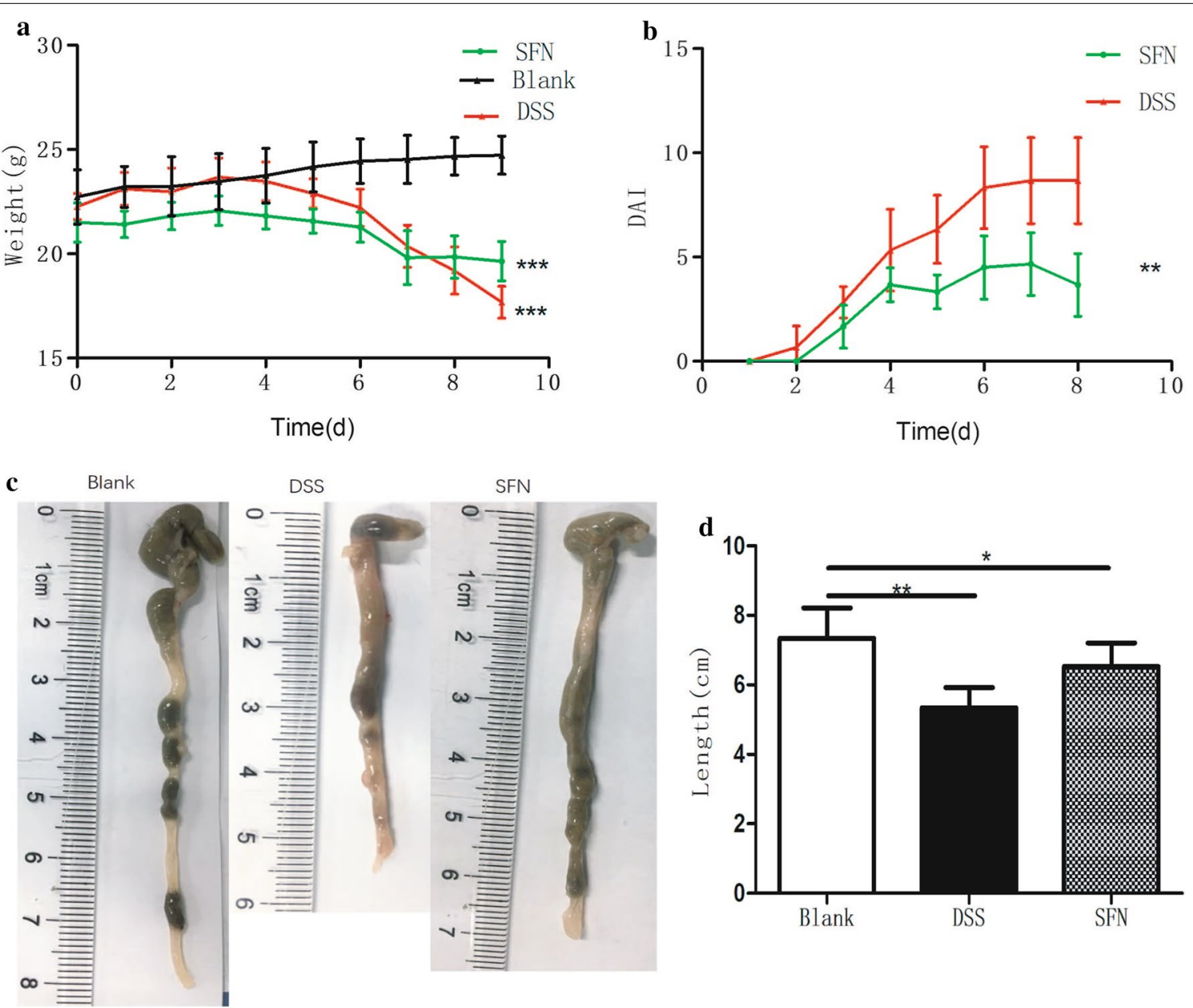

Fig. 1 Effects of Sulforaphane (SFN) on clinical signs in DSS-induced colonitis. ( $n=6$ per group). a DSS-induced ulcerative colitis caused the loss of body weight in mice. $\mathbf{b}$ Disease activity index score in the SFN and DSS groups. $\mathbf{c}$, d Effect of SFN on DSS-induced colon shortening. ${ }^{*} \mathrm{P}<0.05$, ${ }^{* *} \mathrm{P}<0.01,{ }^{* * *} \mathrm{P}<0.001$ 

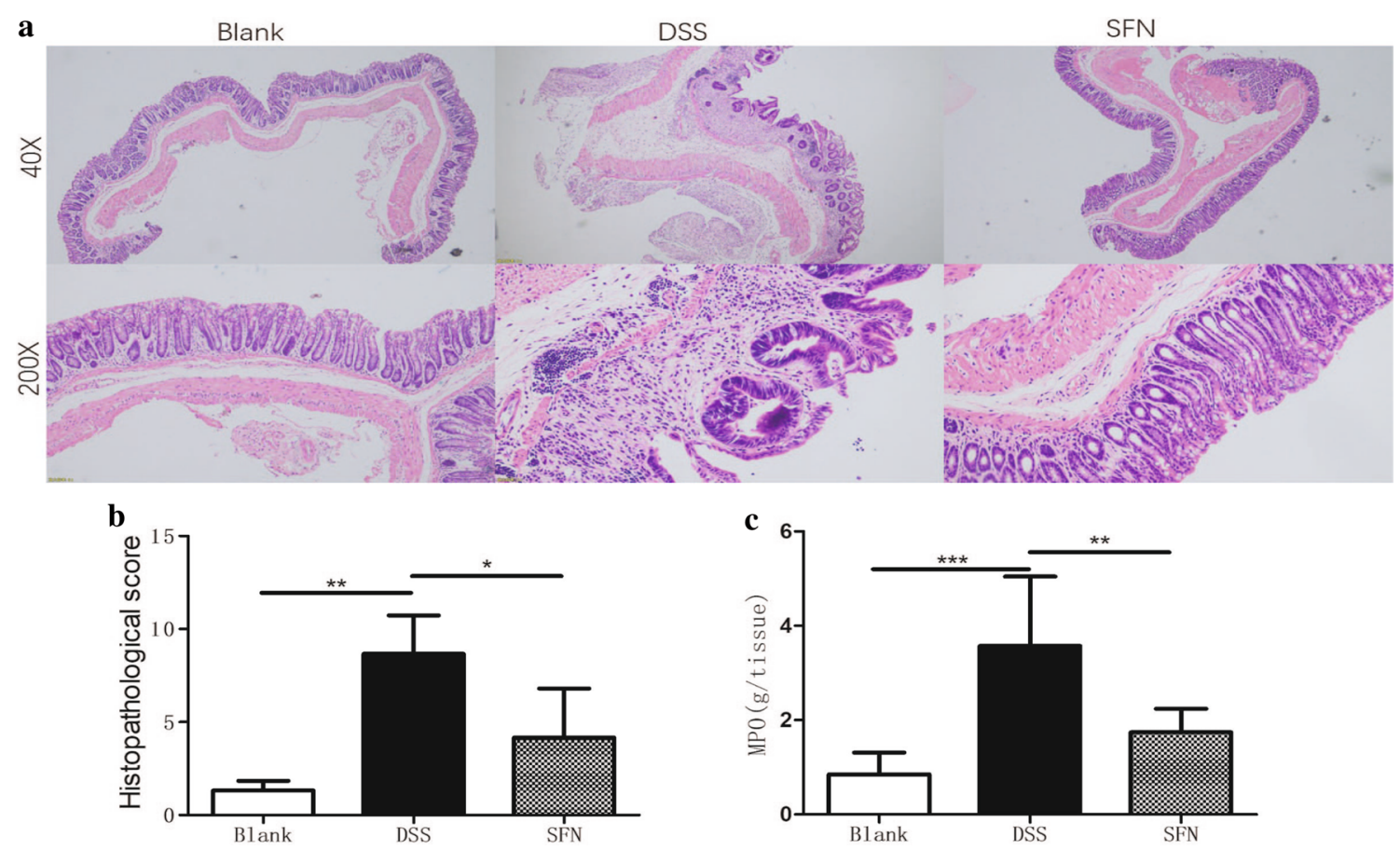

Fig. 2 Sulforaphane (SFN) pretreatment alleviated DSS-induced colon inflammation (a) Histopathological changes after DSS stimulation in colon $(\times 40, \times 200)$. b Effects of SFN on microscopic damage scores. $\mathbf{c}$ Effects of SFN on MPO activity. ${ }^{* P}<0.05,{ }^{* *} P<0.01,{ }^{* * *} P<0.001$

between blank group and DSS group was more statistically significant $(\mathrm{P}<0.01)$.

\section{Sulforaphane reversed DSS-induced gut dysbiosis}

For all the three groups, using Illumina HiSeq/MiSeq platforms sequenced variable regions V4-V5 of the $16 \mathrm{~S}$ rRNA gene from fecal samples. The purpose was to evaluate the changes in the gut microbial community. Operational taxonomic units (OTUs) was uesd to present the results with a $97 \%$ homology cutoff value (Fig. 3). An obvious clustering of the microbiota composition was presented by principal coordinates analysis (PCoA) for all three group (Fig. 3a). The clustering of microbiota composition from PCoA and microbial communities from alpha diversity analysis at the OTU level in DSS group were significantly different from the other two groups. Instead, the SFN group and the blank group had a similar microbial community in the feces (Fig. 3b). The diversity was shown in Shannon index analysis (Fig. 3c-e). The higher value indicated the higher diversity richness. The community richness in the DSS group were lowe than other two groups (Fig. 3d, $\mathrm{P}<0.001$ ).

At the phylum level, the relative abundance of the predominant taxa identified from sequencing of all three groups were shown in Fig. 4, which can present the specific changes in the gut microbiota. Figure 4a illustrated a detailed overview of the intestinal bacteria composition.
In this study, Firmicutes, Bacteroidetes, and Proteobacteria had a higher richness in all samples. Moreover, the three phyla differed wildly in each group. After 7 days, the intestinal bacteria composition of the SFN group had no statistic difference with other two groups. After 14 days, the relative abundances of Firmicutes in the DSS group was higher (Fig. $4 \mathrm{c}, \mathrm{P}<0.05$ ), but which in the SFN group was lower (Fig. 4d, $\mathrm{P}<0.05$ ). The abundance of Bacteroidetes in the DSS group was the lowest, but SFN significantly increased the level of Bacteroidetes(Fig. 4b, $\mathrm{P}<0.05)$. Firmicutes-to-Bacteroidetes ratio was a reliable indicator used herein to assess the gut microbiota. The $F / B$ ratio in the blank group was 0.6469 , and other two groups had an increased ratio. Moreover, the ratio showed siginificant difference between the DSS group and the blank group $(\mathrm{P}<0.05)$.

At the family level, in the first 7 days, the blank group significantly increased the level of Bacteroidaceae, Bacteroidales_S24-7, Lachnospiraceae, Ruminococcaceae, Rikenellaceae and Prevotellaceae, and the SFN group had a similar trend. In the second 7 days, the abundances of Lachnospiraceae, Bacteroidales_S24-7, Rikenellaceae, Lactobacillales and Prevotellaceae was significantly decreased in the DSS group than in the blank group $(\mathrm{P}<0.05)$. In contrast, the abundances of Clostridiaceae_1, Erysipelotrichaceae, Campylobacteraceae, Peptostreptococcaceae and Enterobacteriaceae increased 


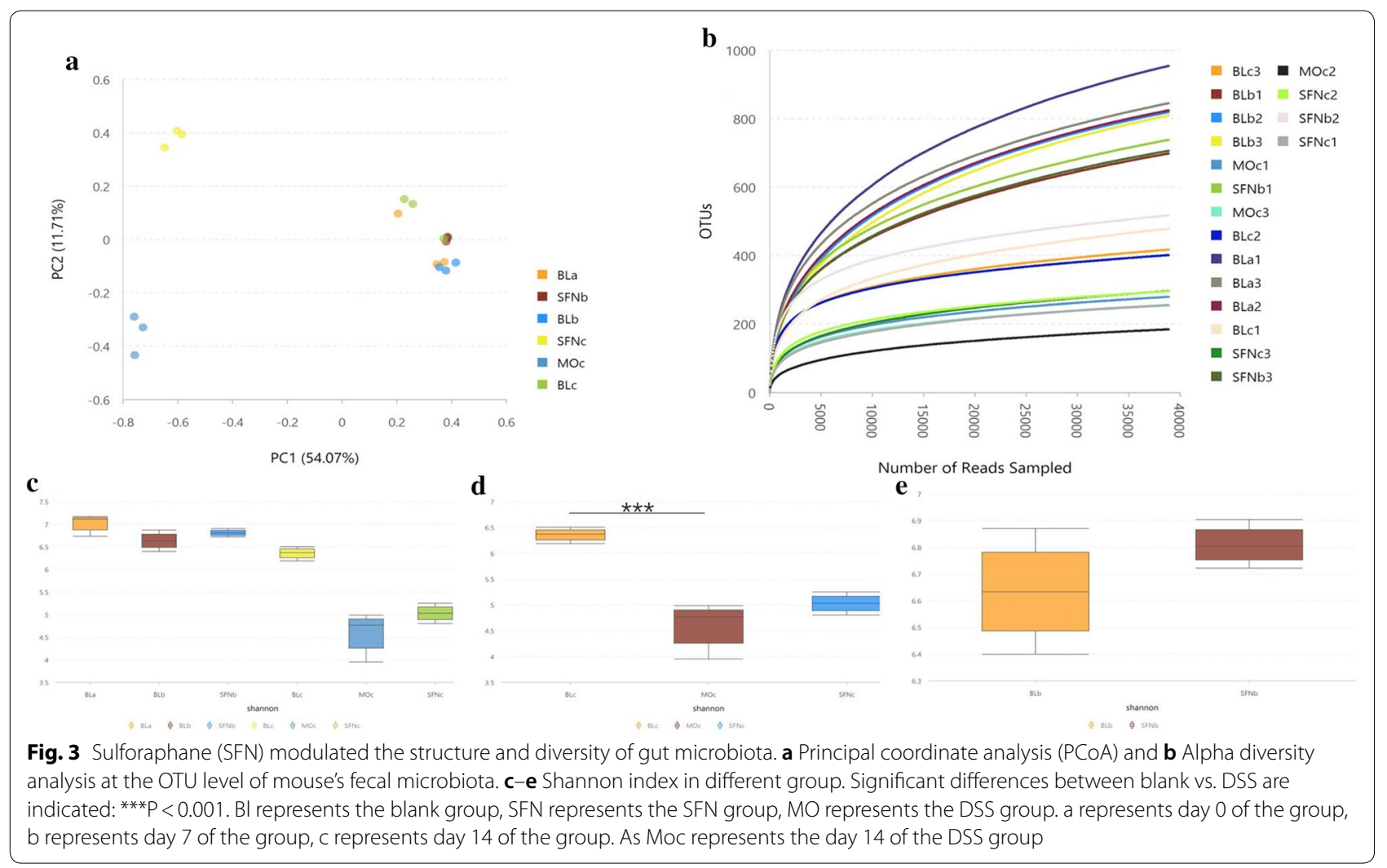

in the DSS group than in the blank group. The SFN had an obvious inhibitory action to the relative abundance of Bacteroidaceae, Enterobacteriaceae, Bacteroidales S24-7, Rikenellaceae and Prevotellaceae. Overall, these results indicated that SFN could prevent the gut dysibosis induced by DSS in mice.

\section{Sulforaphane would shift the balance to Butyricicoccus on inflammation}

In a further step, to identify the faecal microbiota meaningful changes among the three groups, the relative abundance of 36 genera were presented by branch diagram through Lefse analysis (Fig. 5). Obviously, different groups showed different genera levels. Alloprevotella belongs to Bacteroidea, Lachnospiraceae NA4A136 group and Roseburia belong to Firmicutes, which are the specific bacteria in the blank group. In the DSS group, Epsilonproteobacteria, Campylobacterales, Campylobacteraceae, Campylobacter and Campylobacter helveticus belong to Proteobacteria, Erysipelotrichia, Erysipelotrichaceae and Turicibacter belong to Firmicutes. Moreover, in the SFN group, Gammaproteobacteria, Enterobacteriales, Enterobacteriaceae, Escherichia Shigella belong to Proteobacteria, while Bacilli, Lactobacillaceae, Peptostreptococcaceae, Clostridium sensu stricto 1 belong to Firmicutes.
In order to further analyze the specific bacteria at the genus level, the metastats method was used to compare the bacteria between the DSS group and the blank group, and seven bacteria with statistical difference were selected $(P<0.01)$. In the blank group, the relative abundances of Ruminiclostridium6, Alloprevotella, Butyricicoccus, Lachnospiraceae_UCG-006 and Family_XIII_UCG -001 were increased. On the contrary, the relative abundance of Turicabaracter was reduced. Compared with the DSS group, the relative abundance of Butyricicoccus in the SFN group increased $(P=0.0337)$, while that of Turibaccharacter decreased $(\mathrm{P}=0.0056)$.

To further study, the quantity of Butyricicoccus gene was detected by fluorescence. The quantity of Butyricococcus in the DSS group is much lower than the quantity in the blank group (Table $1, \mathrm{P}<0.05$ ). The quantity of Butyricicoccus in SFN group was higher than that of the DSS group, but less than that of the blank group. SFN could reverse the changes of Butyricicoccus to the DSS group at the genus level.

\section{Discussion}

Ulcerative colitis is one common chronic diseases of digestive system. At present in clinical, doctors use antiinflammatory drugs or immunosuppressive drugs to treat UC. However, these drugs usually cause some side 

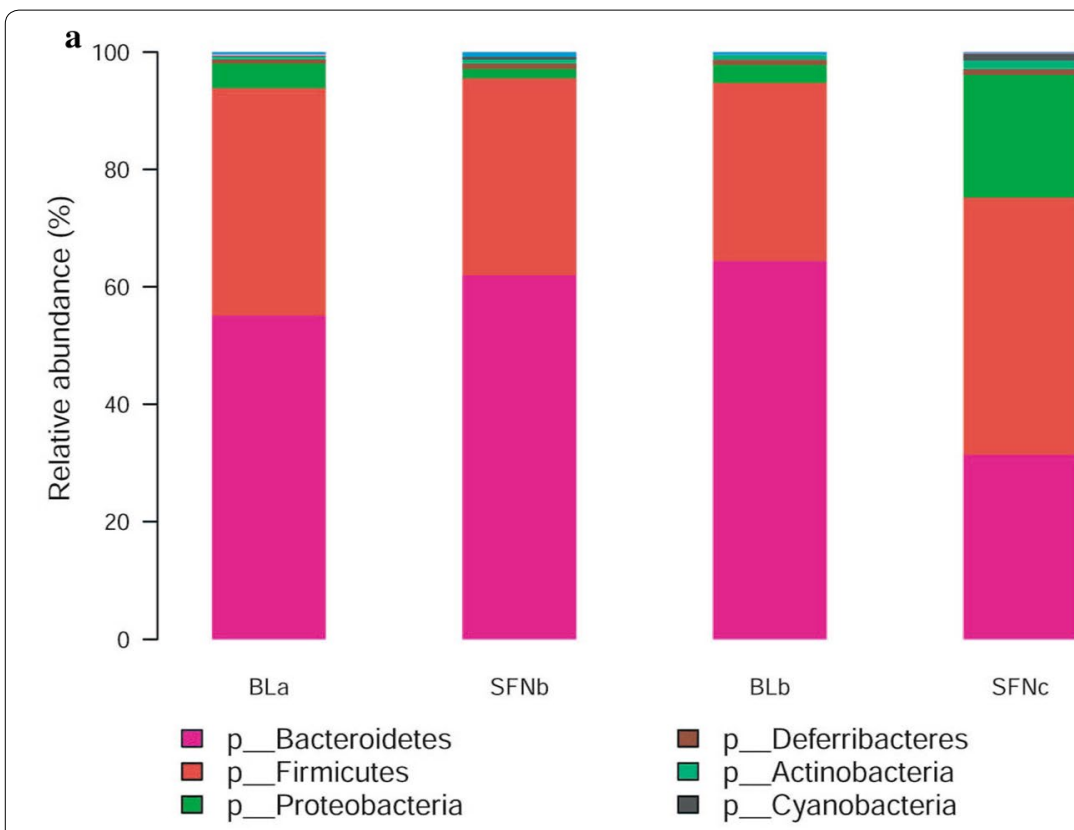

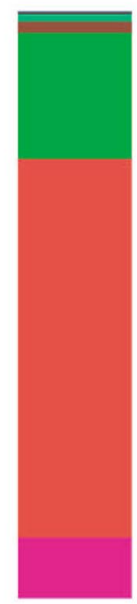

MOc

$\square \quad$ p__ Verrucomicrobia $\square$ Other b

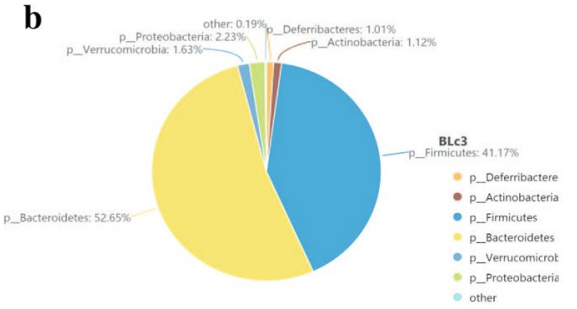

c

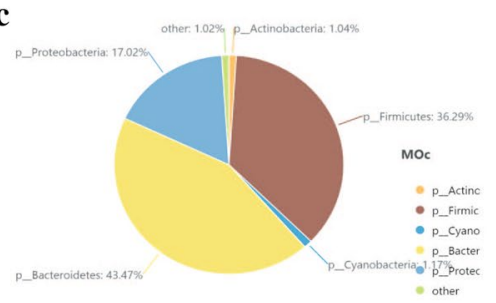

d

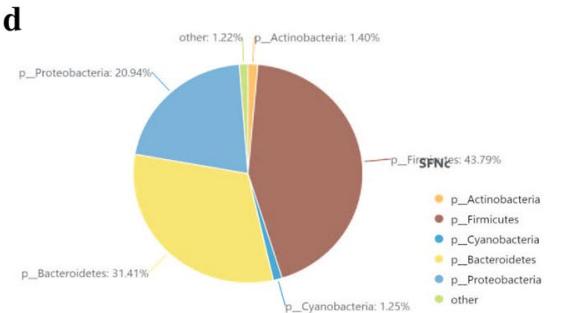

Fig. 4 SFN modulated the composition of gut microbiota at the phylum level. a Phylum-level taxonomic distributions of the microbial communities in feces. $\mathbf{b}-\mathbf{d}$ Composition of the phylum level in the 14th day in three groups

effects. UC has a long course of disease, which is easy to recur, and cannot maintain long-term clinical remission. The need of new treatment methods is urgent.

SFN is a kind of natural isothiocyanate, which exists in cruciferous plants, such as cabbage and broccoli. SFN can activate the nuclear factor E2 related factor 2 (Nrf2) pathway, and induce the body to produce type II detoxification enzymes, which have antioxidant, anti-cancer activity and immune regulation functions (Sun et al. 2015). In recent years, SFN has also been found to have good anti-inflammatory and antibacterial effects. Wagner (Wagner et al. 2013) showed that SFN pretreatment had a protection on DSS-induced colitis by reducing the expression of inflammatory biomarkers in intestinal mucosa and increasing the expression of Nrf2 dependent genes. Morever, Hubbard (Hubbard et al. 2017) suggested that the broccoli riched in SFN affect the structure of intestinal microbial community and attenuate colitis by alylhydrogen receptor (AHR) dependent mode in $\mathrm{Ahr}^{\mathrm{b} / \mathrm{b}}$ mice. At present, there is no study on the effect of SFN on the intestinal flora of UC mice. Therefore, the purpose of this study is to find out the effect of SFN on the intestinal microbial community of UC mice.

DSS-induced UC in mice is one of the most mature methods at present. The influencing factors of DSS modeling include: molecular weight and concentration of DSS, research environment, mouse species, administration time, etc. (Kitajima et al. 2000). After 7 days of DSS intervention, the mice in the DSS group lost weight, shortened the length of blood stool and colon, and significantly increased the DAI score. The DAI score, colon length and histopathological score in the SFN group were all improved $(\mathrm{P}<0.05)$ than that in the DSS group. The MPO activity of the SFN group was significantly lower than that of the DSS group $(\mathrm{P}<0.05)$, which indirectly proved that SFN reduced the inflammatory degree of acute ulcerative colitis in mice.

Dysbacteriosis of intestinal flora is intimately correlated to the pathogenesis of DSS-induced UC (Chen et al. 2016). The results showed that, compared with the blank group, after 7 days of DSS intervention, the Shannon index of intestinal microflora in other two groups 


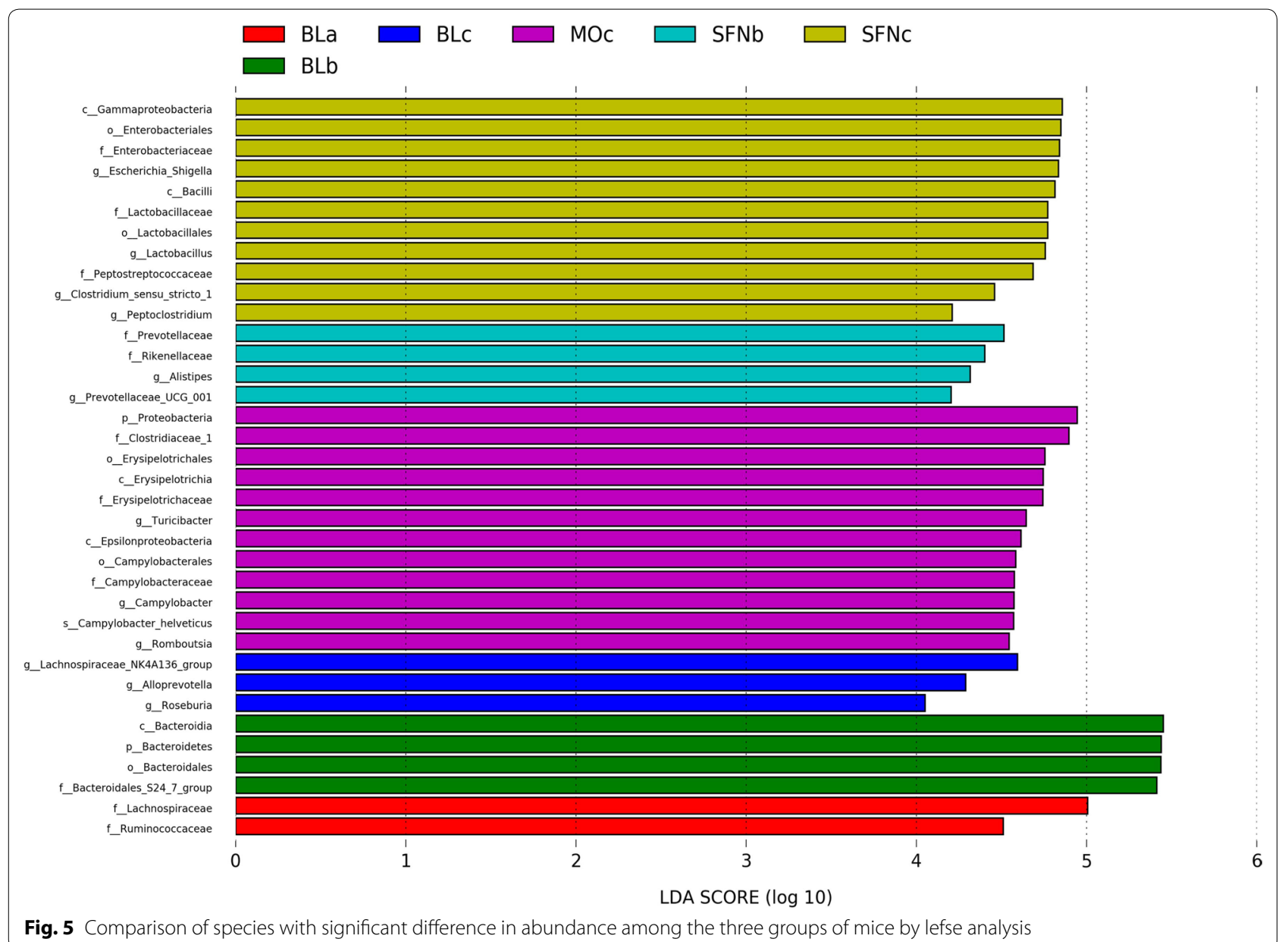

Table 1 Quantitative analysis results of Butyricicoccus

\begin{tabular}{lrrrc}
\hline Sample name & Copies & Copies & Copies & Average copies \\
\hline SFNC & 4.8709 & 10.7772 & 40.1199 & 18.5893 \\
MOC & 12.0912 & 1.5708 & 5.5293 & 6.4171 \\
BLC & 2847.3337 & 66.2252 & 72.8111 & 995.4567 \\
\hline
\end{tabular}

descended, that is, the diversity of microflora decreased. PCoA analysis showed that the distance between the blank group and the DSS group was far from each other, indicating that there was a great difference in the composition of the flora between the two groups, which was same with the previous conclusion of Yang et al. (2017). The DSS intervention could change the $\beta$ diversity of the intestinal microbial community in mice, that is, change the species composition.

According to the results of phylum level, the three major phyla were Firmicutes, Proteobacteria and Bacteroidetes in the three groups. In comparision to the blank group, the proportion of Firmicutes and Proteobacteria in the DSS group grown. Meanwhile, the proportion of Bacteroidetes and Verrucomibia dropped, and the ratio of $F / B$ grown $(\mathrm{P}<0.05)$. In comparison to the DSS group, SFN group had lower Firmicutes, higher Bacteroides and lower $F / B(\mathrm{P}<0.05)$. There was no obviously difference in $F / B$ between SFN group and blank group. Wu et al. (2016) showed that during AOM/DSS induced colitis related colon cancer in mice, the level of Bacteroides decreased and that of Firmicutes increased, which was consistent with the results of this experiment. However, Yeom et al. (2016) showed that after DSS intervention, the proportion of Bacteroides in mouse intestinal microflora increased, while that of Firmicutes decreased. Some studies have suggested that the increase of $F / B$ ratio, namely, the increase of Firmicutes, and the decrease of Bacteroides have a protective effect on inflammatory bowel disease. There are two reasons for the difference: First, the intestinal flora of mice can be affected by many factors such as feed, drinking water, environment and so on. At the same time, the structure of intestinal flora is 
intimately correlated to the disease factors such as the course of ulcerative colitis, the location of lesions, the degree of inflammation and so on. Secondly, in this study, the number of mice in each group is less, and the individual differences are larger. Therefore, the detection of intestinal flora is also biased.

According to the results of family level, after DSS modeling, in comparision to the blank group, the bacterial of DSS-induced mice changed dramatically. However, the intestinal flora of SFN group was closer to that of blank group. In comparison to the DSS group, Erysipelototrichaeae and Campylobacteraceae was found significantly decreased in the blank and SFN group. While the relative abundance of Bacteroidales_S24-7, Rikenellaceae and Prevotellaceae increased $(\mathrm{P}<0.05)$. According to relevant research reports, Bacteroidales_S24-7 is a kind of intestinal probiotics, which is negatively related to intestinal inflammation. Rikenellaceae is a hydrogen producing bacterium, which can selectively neutralize cytotoxic reactive oxygen species (ROS) and protect cells from oxidative stress, thus improving the symptoms of inflammatory bowel disease (Rooks et al. 2014). In general, Bacteroidales_S24-7 and Rikenellaceae are more likely to have beneficial bacteria and protect the intestine. The role of Prevotellaceae is unclear. On the one hand, it has been reported that Prevotellaceae is prominent in IBD patients. Wright et al. (2000) suggested that Prevetelaceae may damage the function of intestinal mucosal barrier by producing sulfatase which can degrade mucopolysaccharide, which is increased in IBD patients' intestinal biopsies. Prevotellaceae may also lead to the deterioration of chronic intestinal inflammation in IBD mice. On the other hand, Monk et al. (2016) showed that cranberry bean rich diet can reduce the symptoms of ulcerative colitis in mice, and beans can increase the abundance of Prevetelaceae and Bacteroidales_S24-7 in mice intestine. De Cruz et al. (2015) proved that Prevetellaceae is a kind of intestinal microorganism which has relation to the remission of inflammatory bowel disease. Therefore, the role of Prevotellaceae needs further study in the future.

Currently, in antiretroviral treatment of patients infected with human immunodeficiency virus, it has been proved that Erysipelototrichaeae is associated with elevated TNF levels and chronic intestinal inflammation (Dinh et al. 2015). Hubbard et al. (2017) study found that eating broccoli can degrade the relative abundance of Erysipelototrichaeae in the intestine of colitis mice and alleviated colitis. Campylobacteraceae belongs to Proteobacteria. Many Campylobacter species may be relative to the pathogenesis of IBD. A meta-analysis by Natalia (Castano-Rodriguez et al. 2017) showed that Campylobacter (especially C. showae and C. concisus) raise the chance of IBD. The results of Lefse analysis of mouse intestinal flora showed that Erysipelototrichaeae and Campylobacteraceae is also a biomarker in the DSS group. The intervention of SFN can reduce the relative abundance of the two bacteria.

In order to further analyze the specific strains, seven specific strains were found out by comparing the intestinal flora of mice in the blank group and the DSS group on the 14th day of the experiment with the metastats method, the specific species were Ruminiclostridium, Alloprevotella, Turicibacter, Butyricicoccus, Lachnospiraceae_UCG-006, Ruminococcaceae_UCG-013 and Family_XIII_UCG-001 $(\mathrm{P}<0.05)$. The relative abundance of Butyricicoccus in the DSS group was the lowest among the three groups, while the relative abundance of Turicabaracter was the highest. Turiribcharacter belongs to Erysipelototrichaeae. Previous studies have shown that the abundance of Tricibacter in the gastrointestinal tract of colitis mice (DSS induced mice and IL-22 deficient mice) is decreased (Zenewicz et al. 2013; Collins et al. 2014). More recently, it has been introduced that in AOM/DSS induced colitis related colon cancer, the relative abundance of Turicabaracter increased (Wu et al. 2016). In the pathogenesis of human ileal pouch inflammation, which previously suffered from UC, Turicabaracter is closely related to the well-known pathogenic bacterium Clostridium perfringens (Falk et al. 2007). Therefore, it is believed that Turibacharacter has two factors affect with the pathogenesis of mammalian immune system and IBD.

Butyricococcus is a Clostridial cluster IV that produces butyrate, and its number is reduced in feces of patients with ulcerative colitis (Falk et al. 2007). Butyrate, a short chain fatty acid, was generated in the fermentation of dietary fiber in colon. Butyrate is not only the main energy source of colonic cells, but also maintains colonic homeostasis by regulating various cell functions, including proliferation, differentiation, apoptosis and control of intestinal epithelial permeability (Hamer et al. 2008). In addition, butyrate is also an effective anti-inflammatory medium, which can promote the function of epithelial barrier, induce the ability of colon regulatory $\mathrm{T}$ cell differentiation, and inhibit the expression of cytokines (Hamer et al. 2008). In this study, RT-PCR was used to present quantitative analysis. The number of Butyricicoccus in the SFN group was more than that in the DSS group, but there was no statistical difference between the two groups. But there was a statistical difference of the number of Butyricoccus in the blank group, which was much higher than that in the other two groups. According to the results, the relative abundance of microbial species altered by SFN treatment showed the difference of gut bacterial compositions compared to the DSS group. SFN has the protective effect on intestine by Butyricococcus. 
There are many bacteria can produce Butyrate, such as Clostridium cluster IV and XIVa bacteria. In this study, only one of them was detected, so the effect of other butyrate producing bacteria on intestinal protection could not be completely denied. In the future experiments, the alleviative effect of SFN on colitis in mice can be further explored.

\section{Acknowledgements}

Not applicable.

\section{Authors' contributions}

YZ and ZYZ designed the study. YZ, LXT, CL, HW and DR performed the actual laboratory analyses. LXT, CL, HW and DR obtained the samples and analyzed the data. YZ and ZYZ wrote the manuscript. All authors read and approved the final manuscript.

\section{Funding}

This work was supported by the National Natural Science Foundation of China (No. 81700458);

\section{Availability of data and materials}

Data will be made available through publication and SRA database (PRJNA611676).

\section{Ethics approval and consent to participate}

The study was conducted under the Guide for the Care and Use of Laboratory Animals (USA) and approved by the Institutional Animal Care and Use Committee of Nanjing Medical University.

\section{Consent for publication}

Not applicable.

\section{Competing interests}

The authors declare that they have no competing interests.

\section{Author details}

1 Department of Gastroenterology, Nanjing First Hospital, Nanjing Medical University, 68 Changle Road, Nanjing 210006, China. ${ }^{2}$ Department of Gastroenterology, The Second Hospital of Nanjing, Nanjing University of Chineses Medicine, Nanjing 210003, China.

Received: 28 February 2020 Accepted: 23 June 2020

Published online: 03 July 2020

\section{References}

Castano-Rodriguez N, Kaakoush NO, Lee WS, Mitchell HM (2017) Dual role of Helicobacter and Campylobacter species in IBD: a systematic review and meta-analysis. Gut 66:235-249

Chen S, Cheng H, Wyckoff KN, He Q (2016) Linkages of Firmicutes and Bacteroidetes populations to methanogenic process performance. J Ind Microbiol Biotechnol 43:771-781

Collins JW, Chervaux C, Raymond B, Derrien M, Brazeilles R, Kosta A, Chambaud I, Crepin VF, Frankel G (2014) Fermented dairy products modulate Citrobacter rodentium-induced colonic hyperplasia. J Infect Dis 210:1029-1041

de Cruz P, Kang S, Wagner J, Buckley M, Sim WH, Prideaux L, Lockett T, Mcsweeney C, Morrison M, Kirkwood CD, Kamm MA (2015) Association between specific mucosa-associated microbiota in Crohn's disease at the time of resection and subsequent disease recurrence: a pilot study. $J$ Gastroenterol Hepatol 30:268-278

Dinh DM, Volpe GE, Duffalo C, Bhalchandra S, Tai AK, Kane AV, Wanke CA, Ward HD (2015) Intestinal microbiota, microbial translocation, and systemic inflammation in chronic HIV infection. J Infect Dis 211:19-27

Falk A, Olsson C, Ahrne S, Molin G, Adawi D, Jeppsson B (2007) Ileal pelvic pouch microbiota from two former ulcerative colitis patients, analysed by DNA-based methods, were unstable over time and showed the presence of Clostridium perfringens. Scand J Gastroenterol 42:973-985
Goto Y, Kurashima Y, Kiyono H (2015) The gut microbiota and inflammatory bowel disease. Curr Opin Rheumatol 27:388-396

Guinane CM, Cotter PD (2013) Role of the gut microbiota in health and chronic gastrointestinal disease: understanding a hidden metabolic organ. Therap Adv Gastroenterol 6:295-308

Hamer HM, Jonkers D, Venema K, Vanhoutvin S, Troost FJ, Brummer RJ (2008) Review article: the role of butyrate on colonic function. Aliment Pharmacol Ther 27:104-119

Hubbard TD, Murray IA, Nichols RG, Cassel K, Podolsky M, Kuzu G, Tian Y, Smith P, Kennett MJ, Patterson AD, Perdew GH (2017) Dietary broccoli impacts microbial community structure and attenuates chemically induced colitis in mice in an Ah receptor dependent manner. J Funct Foods 37:685-698

Kitajima S, Takuma S, Morimoto M (2000) Histological analysis of murine colitis induced by dextran sulfate sodium of different molecular weights. Exp Anim 49:9-15

Liu YC, Hsieh CW, Weng YC, Chuang SH, Hsieh CY, Wung BS (2008) Sulforaphane inhibition of monocyte adhesion via the suppression of ICAM-1 and NF-kappaB is dependent upon glutathione depletion in endothelial cells. Vascul Pharmacol 48:54-61

Monk JM, Lepp D, Zhang CP, Wu W, Zarepoor L, Lu JT, Pauls KP, Tsao R, Wood GA, Robinson LE, Power KA (2016) Diets enriched with cranberry beans alter the microbiota and mitigate colitis severity and associated inflammation. J Nutr Biochem 28:129-139

Ng SC, Bernstein CN, Vatn MH, Lakatos PL, Loftus EV, Tysk C, Morain C, Moum B, Colombel JF (2013) Epidemiology and natural history task force of the international organization of inflammatory bowel Geographical variability and environmental risk factors in inflammatory bowel disease. Gut 62:630-649

Paturi G, Mandimika T, Butts CA, Zhu S, Roy NC, Mcnabb WC, Ansell J (2012) Influence of dietary blueberry and broccoli on cecal microbiota activity and colon morphology in mdr1a (-/-) mice, a model of inflammatory bowel diseases. Nutrition 28:324-330

Pei LY, Ke YS, Zhao HH, Wang L, Jia C, Liu WZ, Fu QH, Shi MN, Cui J, Li SC (2019) Role of colonic microbiota in the pathogenesis of ulcerative colitis. BMC Gastroenterol 19:10

Rooks MG, Veiga P, Wardwell-Scott LH, Tickle T, Segata N, Michaud M, Gallini CA, Beal C, van Hylckama-Vlieg JE, Ballal SA, Morgan XC, Glickman JN, Gevers D, Huttenhower C, Garrett WS (2014) Gut microbiome composition and function in experimental colitis during active disease and treatment-induced remission. ISME J 8:1403-1417

Sun CC, Li SJ, Yang CL, Xue RL, Xi YY, Wang L, Zhao QL, Li DJ (2015) Sulforaphane attenuates muscle inflammation in dystrophin-deficient mdx mice via NF-E2-RELATED Factor 2 (Nrf2)-mediated inhibition of NFkappaB signaling pathway. J Biol Chem 290:17784-17795

Wagner AE, Will O, Sturm C, Lipinski S, Rosenstiel P, Rimbach G (2013) DSSinduced acute colitis in C57BL/6 mice is mitigated by sulforaphane pre-treatment. J Nutr Biochem 24:2085-2091

Wright DP, Rosendale DI, Robertson AM (2000) Prevotella enzymes involved in mucin oligosaccharide degradation and evidence for a small operon of genes expressed during growth on mucin. FEMS Microbiol Lett 190:73-79

Wu M, Wu Y, Deng B, Li J, Cao H, Qu Y, Qian X, Zhong G (2016) Isoliquiritigenin decreases the incidence of colitis-associated colorectal cancer by modulating the intestinal microbiota. Oncotarget 7:85318-85331

Yang Y, Chen G, Yang Q, Ye J, Cai X, Tsering P, Cheng X, Hu C, Zhang S, Cao P (2017) Gut microbiota drives the attenuation of dextran sulphate sodiuminduced colitis by Huangqin decoction. Oncotarget 8:48863-48874

Yeom Y, Kim BS, Kim SJ, Kim Y (2016) Sasa quelpaertensis leaf extract regulates microbial dysbiosis by modulating the composition and diversity of the microbiota in dextran sulfate sodium-induced colitis mice. BMC Complement Altern Med 16:481

Zenewicz LA, Yin X, Wang G, Elinav E, Hao L, Zhao L, Flavell RA (2013) IL-22 deficiency alters colonic microbiota to be transmissible and colitogenic. J Immunol 190:5306-5312

\section{Publisher's Note}

Springer Nature remains neutral with regard to jurisdictional claims in published maps and institutional affiliations. 